\title{
Nicht-invasive lokalisierte Protonen-Magnetresonanz-Spektroskopie in der präoperativen Abklärung medikamentenresistenter Epilepsie
}

\author{
Duc, C O ; Weber, O M ; Meier, D ; Wieser, H G ; Boesiger, P
}

DOI: https://doi.org/10.1515/bmte.1996.41.s1.230

Posted at the Zurich Open Repository and Archive, University of Zurich ZORA URL: https://doi.org/10.5167/uzh-155078

Journal Article

Published Version

Originally published at:

Duc, C O; Weber, O M; Meier, D; Wieser, H G; Boesiger, P (1996). Nicht-invasive lokalisierte ProtonenMagnetresonanz-Spektroskopie in der präoperativen Abklärung medikamentenresistenter Epilepsie. Biomedizinische Technik. Biomedical engineering, 41(s1):230-231.

DOI: https://doi.org/10.1515/bmte.1996.41.s1.230 


\title{
Nicht-invasive lokalisierte Protonen-Magnetresonanz-Spektroskopie
}

\section{in der präoperativen Abklärung medikamentenresistenter Epilepsie}

\author{
C.O. Duc, O.M. Weber, D. Meier, H.G. Wieser" ${ }^{\#}$ P. Boesiger \\ Institut für Biomedizinische Technik und Medizinische Informatik der Universität und ETH Zürich, Schweiz \\ ${ }^{\#}$ Institut für Neurologie, Universitätsspital Zürich, Schweiz
}

\section{EINLEITUNG}

Epilepsien gehören weltweit zu den häufigsten Erkrankungen des menschlichen Nervensystems [1]. Etwa ein Drittel der Patienten spricht auf konventionelle antiepileptische Medikamente nicht (in befriedigender Weise) an, so dass eine operative Entfernung des epileptischen Herdes in Betracht zu ziehen ist. Zur Lokalisierung des epileptischen Fokus gilt nach wie vor das Elektroenzephalogramm (EEG) als Standardmethode. Allerdings müssen zur Detektion von Spikes in tiefer liegenden Hirnregionen die Elektroden operativ in das vermutlich erkrankte Gebiet implantiert werden, was mit nicht unerheblichen Risiken verbunden ist. Weitere häufig zur Konsultation beigezogene Methoden sind die ProtonenEmissions-Tomographie (PET), mit welcher z.B. der Glukoseverbrauch des Gehirns abgebildet werden kann, oder die Single-Photon-Emissions-Computertomographie (SPECT), welche eine Abschätzung der Neurotransmittorrezeptorendichte erlaubt. Bei diesen Methoden wird der Körper jedoch radioaktiver Strahlung ausgesetzt.

Im Gegensatz zu den oben erwähnten Methoden erlauben Magnetresonanztechniken eine Charakterisierung von Gewebseigenschaften ohne jeglichen operativen Eingriff und ohne Belastung durch Röntgenbestrahlung. Während die konventionelle MR-Bildgebung (MRI) vor allem Aufschluss über strukturelle Eigenschaften oder Veränderungen des Gewebes gibt, erlaubt die nicht-invasive Protonen-Magnetresonanz-Spektroskopie $\left({ }^{1} \mathrm{H}\right.$ MRS) eine spezifische und quantitative Erfassung von Stoffwechselprodukten in definierten Regionen des menschlichen Gehirns in vivo. Sie eignet sich daher vorzüglich zur Untersuchung neurodegenerativer Prozesse und zur Langzeitverfolgung beispielsweise von therapeutischen Effekten.

Eingehende präoperative Abklärungen sind von entscheidender Bedeutung für das Gelingen des klinischen Eingriffes in das menschliche Gehim. Bei der Temporallappenepilepsie (TLE), dem häufigsten Epilepsietypen $[1,2]$, ist mehrheitlich der Hippokampus als epileptogene Zone aktiv. Wenn die epileptischen Entladungen nur von der einen Seite ausgehen, besteht die Möglichkeit, durch selektive operative Entfernung des betreffenden Hippokampus (sog. Amygdala-Hippokampektomie [3], AHX) vollständige Anfallsfreiheit zu erlangen. Dabei sind neurologische Defizite minimal, wenn der kontralaterale Hirnteil die Funktionen aufrecht erhalten kann.
Die Möglichkeit, mittels der nicht-invasiven, lokalisierten in vivo Protonenspektroskopie spezifischen Aufschluss über degenerative Erscheinungen im Hippokampus von TLE-Patienten zu erhalten, wurde in einer korrelativen Studie evaluiert. Hierzu wurden die spektroskopischen Daten der pathologischen (ipsilateralen) Region mit dem kontralateralen Bereich in Patienten sowie mit den Werten von gesunden Probanden verglichen, ferner mit den Befunden zur Verfügung stehender (konventioneller) Alternativmethoden (EEG, PET, SPECT, MRI, neurophysiologische Tests) und allenfalls vorhandenen histologisch/histochemischen Analysen operativ entnommener Proben.

\section{METHODEN}

Die MRS-Untersuchungen wurden auf einem klinischen 1.5T Ganzkörpertomographen (Philips Gyroscan ACSII) mit aktiv abgeschirmter Gradientenkopfspule durchgeführt. Anhand T2-gewichteter MR-Übersichtsbilder werden die spektroskopischen Volumen (2-3ml) derart positioniert, dass sie die Hippokampusregion möglichst einschliessen, aber keine Ventrikelbeiträge enthalten (Bild 1a,b). Zur Lokalisierung wurde die Doppel-SpinEcho-Sequenz PRESS mit vorangehender selektiver Unterdrückung des Wassersignals (CHESS) verwendet. Pro Spektrum wurden Signale über ca. 10min bei einer Repetitionszeit (TR) von 6s gemittelt. Wenn möglich wurden die Spektren nacheinander bei langer (136ms) und kurzer (30ms) Echozeit in beiden Hirnhälften aufgenommen. Die Bestimmung der absoluten Metabolitenkozentrationen erfolgte mittels der Simulationsphantom-Methode, welche in einem anderen Beitrag dieses Kongressbandes beschrieben ist. Hierbei wird die Messung an einem Kalibrationsphantom, welches den menschlichen Kopf simuliert und eine Eichlösung enthält, mit denselben, optimierten Messparametern wiederholt (Figs. 1a-c).

68 Patienten (28-49j., im Mittel 41j.) mit medikamentenresistenter TLE wurden aufgrund EEG- und neurophysiologischem Gutachten als Kandidaten für eine selektive AHX ausgewählt. Weiterhin beinhaltete die präoperative Abklärung klinisches MRI, FDG-PET und in einigen Fällen SPECT.

21 gesunde Probanden der selben Altersklasse wurden zur Aufnahme von Kontrolldaten mit dem gleichen MRS-Protokoll untersucht. 

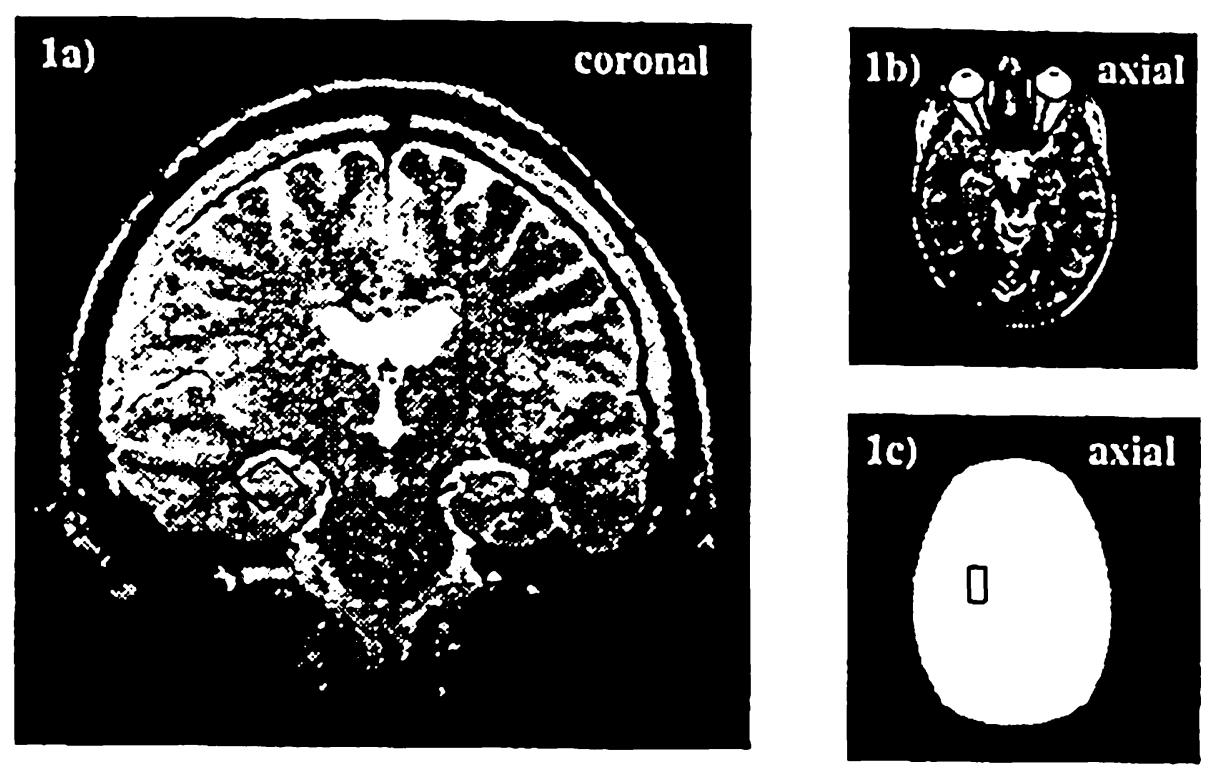

Figs. 1: Das selektierte Volumen zur ${ }^{1} H$ MRS-Messung des Hippokampus in vivo ist auf den MR-Bildern la,b eingezeichnet. Zur Bestimmung der Metabolitenkonzentrationen wird die Messung am Kalibrationsphantom mit den selben Aufnahmeparametern wiederholt (Bild Ic).

\section{ERGEBNISSE}

Spektren, welche im epileptogenen Hippokampus aufgenommen wurden, zeigen charakteristische Veränderungen sowohl in Lang- wie in Kurz-Echozeit-Spektren im Vergleich zur (relativ) gesunden Gegenseite (Figs. 2).

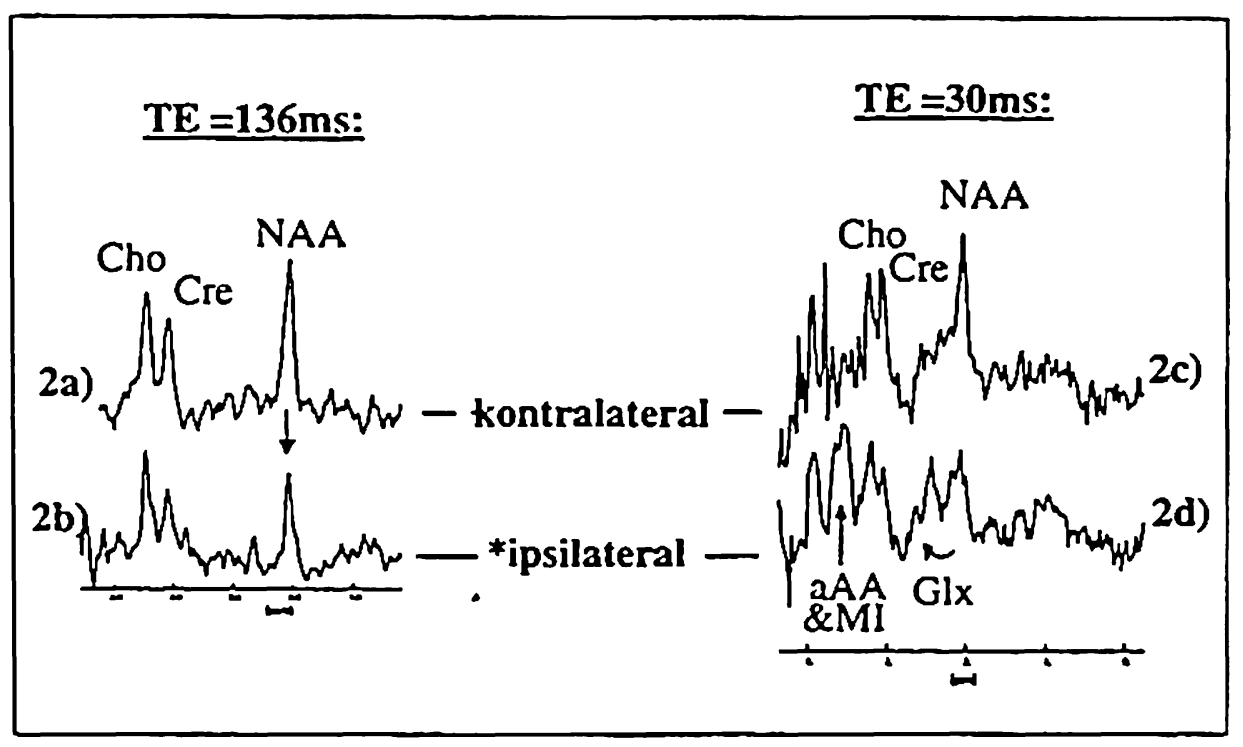

Figs. 2: Hippokampusspektren von TLE-Patienten. Die auf der epileptogenen (*ipsilateralen) Seite aufgenommen Spektren (unten; 2b: TE $=136 \mathrm{~ms}, 2 \mathrm{~d}: T E=30 \mathrm{~ms}$ ) zeigen im Vergleich $z u$ den kontralateralen Aufnahmen (oben; 2a: $T E=136 \mathrm{~ms}, 2 c: T E=30 \mathrm{~ms}$ ) deutliche Unterschiede. Besonders markant ist die Reduktion von NAA (N-AzetylAspartat). NAA gilt als neuronaler Marker und verschwindet mit dem Zugrundegehen von Nervenzellen. Nur in KurzEchozeit-Spektren (2c,d) sind Veränderungen im Bereich von Glutamin/Glutamat (Glx) und Myo-Inositol (MI) detektierbar. Letztere geben Hinweise auf Gliose.

Die absolute Quantifizierung der Stoffwechselprodukte ergab signifikant reduzierte Konzentrationen für NAA und Kreatin auf der epileptogenen Seite; interessanterweise war auch auf der Gegenseite eine im Mittel signifikant reduzierte NAA-Konzentration zu messen, welche etwa zwischen dem Normalwert und dem ipsilateralen pathologischen Wert iiegt (Fig. 3).

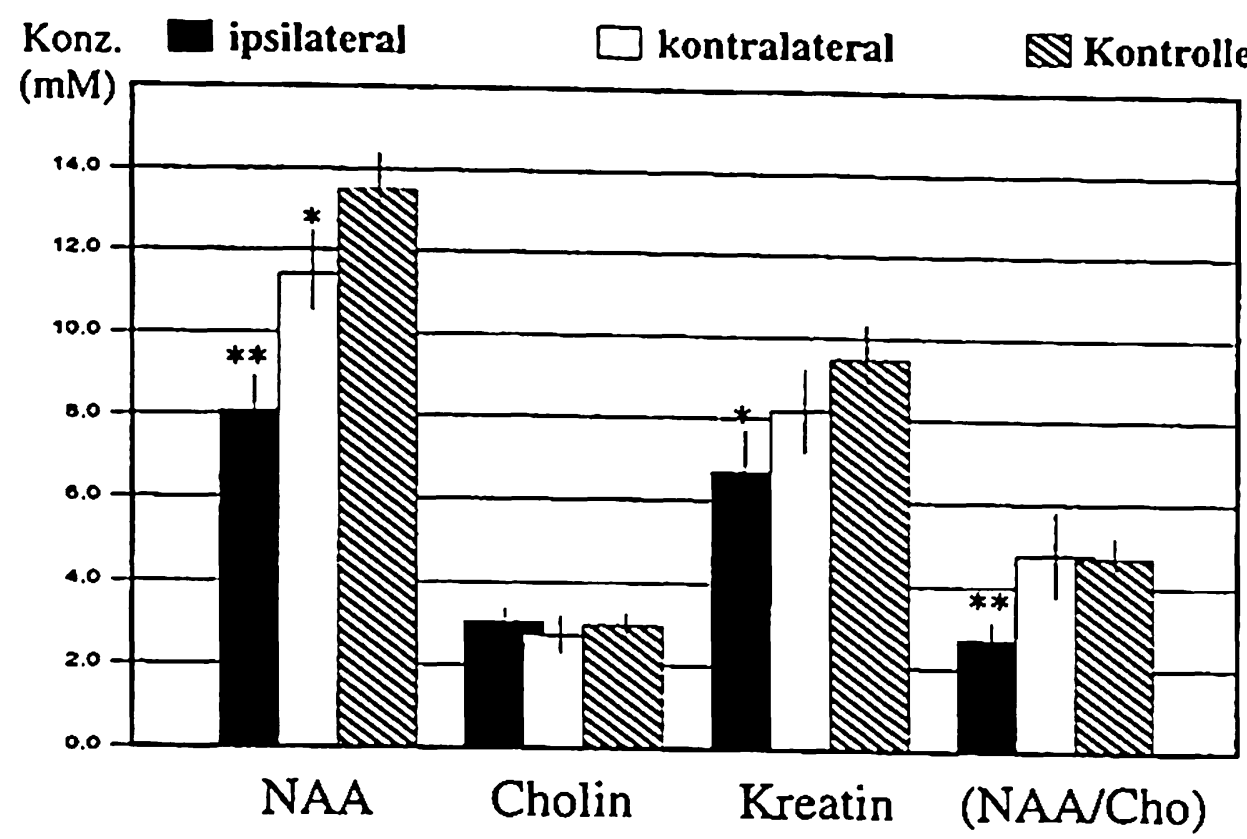

Fig. 3: Der neuronale Marker NAA weist im epileptogenen Hippokampus der Patienten im Vergleich zu den Kontrollen stark erniedrigte Konzentrationswerte auf $\left(p=0.001^{* *}\right)$. Wichtig ist weiterhin die Erkenntnis, dass auch kontralateral zum epileptogenen Fokus die NAAKonzentration bei Patienten signifikant unter dem Normalwert liegt $\left(p<0.03^{*}\right)$.

\section{DISKUSSION}

Die hochsignifikante Konzentrationsabnahme von NAA, einem spezifisch neuronalen Metaboliten [4], im epileptogenen Hippokampus von TLE-Patienten korreliert gut mit den histologischen Berichten über starke Nervendegeneration in operativ entnommen Hippokampusproben [2,5]. Eine Reduktion der NAA-Konzentration auch kontralateral zum (hauptsächlich) erkrankten Hippokampus ist für die präoperative Evaluation von Bedeutung, da für die Aufrechterhaltung gewisser Hirnfunktionen mindestens ein funktioneller Hippokampus noch vorhanden muss.

Auch die häufig beobachteten Veränderungen in KurzEchozeit-Spektren, nämlich erhöhte Signale im Bereich von Myo-Inositol und der Trend zur Verschiebung von Glutamat zu Glutamin und -Derivaten, kann in Übereinstimmung mit dem häufigen Befund von Astrogliose (d.h. einer Vermehrung nicht-neuronaler Hirnzellen) in histologischen Analysen interpretiert werden: sowohl Myo-Inositol wie auch Glutamin (und -Derivate) sind in Gliazellen in höheren Konzentrationen vorhanden [6], während Glutamat in Nervenzellen stärker konzentriert ist .

Die Resultate der volumenselektiven ${ }^{1} \mathrm{H}$ MRS stimmen ferner mit denjenigen der konventionellen Diagnostik überein, was für einen routinemässigen Einsatz dieser nicht-invasiven Methode in der medizinische (präoperative) Abklärung spricht.

\section{LITERATURHINWEISE}

1. K. Masuhr, M. Neumann: Neurologie, Hippokrates Verlag Stuttgar (1992) 2. A.V. Delgado-Escueta et al., Adv. Neurol. 44, 3-55 (1986)

3. H.G. Wieser, Adv. \& Techn. Slandards in Neurosurgery 13, 39-133 (1986)

4. F.A. Howe, J.R. Griffiths et al., Magnet. Reson. Quarerly 9. 31 -59 (1993)

5. C.R. Hauser, Brain Res. 535, 195-204 (1990)

6. J. Peeling and G. Sutherland. Magnet. Reson. Med. 24, 123-136 (1992) 